\title{
Membrane-Associated Glucocorticoid Activity Is Necessary for Modulation of Long-Term Memory via Chromatin Modification
}

\author{
Benno Roozendaal, ${ }^{1}$ Angelina Hernandez, ${ }^{2}$ Sara M. Cabrera, ${ }^{3}$ Roelina Hagewoud, ${ }^{4}$ Melissa Malvaez, ${ }^{2}$ \\ Daniel P. Stefanko, ${ }^{2}$ Jakob Haettig, ${ }^{2}$ and Marcelo A. Wood ${ }^{2}$ \\ ${ }^{1}$ Department of Neuroscience, Section Anatomy, University Medical Center Groningen, University of Groningen, 9713 AV Groningen, The \\ Netherlands, ${ }^{2}$ Center for the Neurobiology of Learning and Memory, Department of Neurobiology and Behavior, University of California, Irvine, \\ California 92697-3800, ${ }^{3}$ Department of Biological Sciences, Allergan, Irvine, California 92612, and ${ }^{4}$ Department of Molecular Neurobiology, \\ University of Groningen, 9751 NN Haren, the Netherlands
}

Glucocorticoid hormones enhance the consolidation of long-term memory of emotionally arousing training experiences. This memory enhancement requires activation of the cAMP-dependent kinase pathway and the subsequent phosphorylation of cAMP responseelement binding (CREB) protein. Here, we demonstrate that glucocorticoids enhance the consolidation of hippocampus-dependent and hippocampus-independent aspects of object recognition memory via chromatin modification. More specifically, systemic corticosterone increases histone acetylation, a form of chromatin modification, in both the hippocampus and insular cortex following training on an object recognition task. This led us to examine whether increasing histone acetylation via histone deacetylase (HDAC) inhibition enhances memory in a manner similar to corticosterone. We found a double dissociation between posttraining HDAC inhibitor infusion into the insular cortex and hippocampus on the enhancement of object recognition and object location memory, respectively. In determining the molecular pathway upstream of glucocorticoids' effects on chromatin modification, we found that activation of membraneassociated glucocorticoid receptors (GRs) and the subsequent interaction between phospho-CREB and CREB-binding protein (CBP) appear to be necessary for glucocorticoids to enhance memory consolidation via chromatin modification. In contrast, mineralocorticoid receptors (MRs) do not appear to be involved. The findings also indicate that glucocorticoid activity has differential influences on hippocampus-dependent and hippocampus-independent components of memory for objects.

\section{Introduction}

Glucocorticoid hormones are known to facilitate the consolidation of long-term memory of emotionally arousing training experiences (de Kloet et al., 1999; McGaugh and Roozendaal, 2002; Sandi and Pinelo-Nava, 2007; Roozendaal et al., 2008). In contrast, glucocorticoids appear not to influence memory consolidation of less arousing or neutral information (Buchanan and Lovallo, 2001; Okuda et al., 2004; Kuhlmann and Wolf, 2006). Findings of experiments exploring the neurobiological basis underlying this selectivity indicate that glucocorticoid effects on memory consolidation depend critically on an interaction with arousal-induced noradrenergic activation in the brain (Quirarte et al., 1997; Roozendaal et al., 1999, 2002, 2006). However, despite

Received Nov. 18, 2009; revised Feb. 13, 2010; accepted Feb. 25, 2010.

This research was supported by the National Science Foundation (Grant IOB-0618211 to B.R.), the National Institute of Mental Health (Grant R01MH081004 to M.A.W.), and the Whitehall Foundation (M.A.W.). We thank Dr. James L. McGaugh for critical reading of the manuscript.

Correspondence should be addressed to either of the following: Benno Roozendaal, Department of Neuroscience, Section Anatomy, University Medical Center Groningen, University of Groningen, Antonius Deusinglaan 1, $9713 \mathrm{AV}$ Groningen, The Netherlands, E-mail: b.roozendaal@med.umcg.nl; or Marcelo A. Wood, Center for the Neurobiology of Learning and Memory, Department of Neurobiology and Behavior, University of California, Irvine, CA 92697-3800, E-mail:mwood@uci.edu.

DOI:10.1523/JNEUROSCI.5717-09.2010

Copyright $\odot 2010$ the authors $\quad 0270-6474 / 10 / 305037-10 \$ 15.00 / 0$ previous evidence that glucocorticoid interactions with the noradrenergic system activate the cAMP-dependent protein kinase (PKA) pathway and increase levels of phosphorylated cAMP responseelement binding protein (phospho-CREB) (Roozendaal et al., 2006), it is not known how this arousal effect influences memory consolidation processes.

One possible mechanism by which glucocorticoid-induced phospho-CREB activation may facilitate memory consolidation is via the recruitment of the transcriptional coactivator and potent histone acetyltransferase CREB-binding protein (CBP). CBP is well known as a coactivator recruited by CREB via an interaction between the serine-133 phosphorylated kinase-inducible domain (KID) of CREB and the KIX domain of CBP (Chrivia et al., 1993). We and others have demonstrated that CBP and histone acetylation are crucial for specific forms of long-lasting hippocampal synaptic plasticity and long-term memory (Guan et al., 2002; Bourtchouladze et al., 2003; Alarcón et al., 2004; Korzus et al., 2004; Wood et al., 2005, 2006) (for review, see Barrett and Wood, 2008). Impairments in CBP activity and decreases in histone acetylation result in deficient synaptic plasticity and memory. In contrast, increasing histone acetylation via histone deacetylase (HDAC) inhibition enhances synaptic plasticity and memory (Alarcón et al., 2004; Levenson et al., 2004; Yeh et al., 
2004; Fischer et al., 2007; Vecsey et al., 2007; Stefanko et al., 2009). The ability of HDAC inhibition to enhance synaptic plasticity in the hippocampus and hippocampus-dependent memory appears to be dependent on the phospho-CREB:CBP interaction (Vecsey et al., 2007). Inducing a histone hyperacetylated state via HDAC inhibition appears to facilitate transcription by relaxing chromatin structure, resulting in enhanced synaptic plasticity and long-term memory processes [see Barrett and Wood (2008) for review on histone acetylation in memory processes].

The current study investigated whether glucocorticoids enhance memory consolidation of hippocampus-dependent and hippocampus-independent aspects of object recognition training via $\mathrm{CBP}$ and histone acetylation. Although our previous studies have demonstrated that both glucocorticoids (Okuda et al., 2004; Roozendaal et al., 2006) and CBP (Wood et al., 2006; Stefanko et al., 2009) are involved in modulating long-term memory of object recognition, no study to date has investigated the feasible link between these two modulatory mechanisms with regard to memory formation. Our findings indicate that activation of membrane-associated glucocorticoid receptors (GRs) and the subsequent interaction between phospho-CREB and CBP are necessary for glucocorticoids to enhance memory consolidation via chromatin modification. However, the findings suggest some intriguing differences between glucocorticoid effects on hippocampus-dependent and hippocampus-independent components of object recognition memory.

\section{Materials and Methods}

Subjects. Male Sprague Dawley rats (360-420 g at time of training) from Charles River Laboratories were individually housed in a temperaturecontrolled $\left(22^{\circ} \mathrm{C}\right)$ vivarium on a $12 \mathrm{~h} / 12 \mathrm{~h}$ light/dark cycle (lights on: 7:00 A.M. to 7:00 P.M.) and given food and water ad libitum. For some experiments, male C57BL/6J mice obtained from The Jackson Laboratory were used. The $C B P^{\mathrm{KIX} / \mathrm{KIX}}$ homozygous knock-in mice were generated as previously described (Kasper et al., 2002). Mice were group housed (4 mice per cage) and maintained on a standard $12 \mathrm{~h} / 12 \mathrm{~h}$ light/dark cycle with ad libitum access to food and water. Training and testing were performed during the light phase of the cycle between 10:00 A.M. and 3:00 P.M. All experimental procedures were in compliance with National Institutes of Health guidelines and were approved by the Institutional Animal Care and Use Committee of the University of California, Irvine.

Object training and testing procedures (rats). The experimental apparatus was an open-field box $(40 \times 40 \times 40 \mathrm{~cm})$ made of gray-painted wood with a floor covered with sawdust, placed in a dimly illuminated room. The objects to be discriminated were white glass light bulbs $(6 \mathrm{~cm}$ diameter, $11 \mathrm{~cm}$ length) and transparent glass vials $(5.5 \mathrm{~cm}$ diameter, $5 \mathrm{~cm}$ height). All rats were handled $1-2 \mathrm{~min}$ for $5 \mathrm{~d}$ immediately preceding the training day. On the training trial, the rat was placed in the experimental apparatus and allowed to explore two identical objects (A1 and A2), placed $5 \mathrm{~cm}$ away from the corners of the apparatus for $3 \mathrm{~min}$. To avoid the presence of olfactory trails, sawdust was stirred and the objects were thoroughly cleaned with $70 \%$ ethanol between rats. Retention was tested $24 \mathrm{~h}$ after the training trial. For object recognition memory (ORM) testing, one copy of the familiar object (A3) and a new object (B) were placed in the same location as stimuli during the training trial. For object location memory (OLM) testing, one copy of the familiar object (A3) was placed in the middle of the box, and the other familiar object (A4) was placed in the same location as during the training trial. All combinations and locations of objects were used in a balanced manner to reduce potential biases due to preference for particular locations or objects. The rat was placed in the experimental apparatus for $3 \mathrm{~min}$, and the time spent exploring each object and the total time spent exploring both objects were recorded. Exploration of an object was defined as pointing the nose to the object at a distance of $<1 \mathrm{~cm}$ and/or touching it with the nose. Turning around, climbing, or sitting on an object was not considered exploration. To analyze cognitive performance, a discrimination index was calculated as the difference in time exploring the novel and familiar object (or location), expressed as the ratio of the total time spent exploring both objects. Rats showing a total exploration time $<10$ s on either training or testing were removed from analyses, because data indicated that such rats do not acquire the task (Okuda et al., 2004).

Object training and testing procedures (mice). Object recognition training and testing procedures for mice were slightly modified as compared to the procedures described above. Before training, mice were handled $1-2 \mathrm{~min}$ for $5 \mathrm{~d}$ and were habituated to the experimental apparatus $3 \mathrm{~min}$ a day for 3 consecutive days in the absence of objects. In contrast to rats, mice must be habituated to the experimental apparatus to learn about the objects during training (see Stefanko et al., 2009). The experimental apparatus used for mice was a white rectangular open field $(30 \times 23 \times$ $21.5 \mathrm{~cm}$ ). During the training trial, mice were placed in the experimental apparatus with two identical objects (either $100 \mathrm{ml}$ beakers, $2.5 \mathrm{~cm}$ diameter, $4 \mathrm{~cm}$ height; or large blue Lego blocks, $2.5 \times 2.5 \times 5 \mathrm{~cm}$ ) and were allowed to explore these objects for either 3 or $10 \mathrm{~min}$. During the $24 \mathrm{~h}$ retention test, mice were placed in the experimental apparatus for 5 min. For object recognition memory, one copy of the familiar object (A3) and a new object (B) were placed in the same location as during the training trial. For object location memory, one copy of the familiar object (A3) was placed in the middle of the box and the other familiar object (A4) was placed in the same location as during the training trial. All combinations and locations of objects were used in a balanced manner to reduce potential biases due to preference for particular locations or objects.

Systemic corticosterone treatment. Corticosterone $(1.0 \mathrm{mg} / \mathrm{kg}$; SigmaAldrich) was given subcutaneously immediately after the training trial in a volume of $2.0 \mathrm{ml} / \mathrm{kg}$ body weight for rats and in a volume of $10.0 \mathrm{ml} / \mathrm{kg}$ body weight for mice. Corticosterone was dissolved in 5\% ethanol in saline. This dose of corticosterone is known to induce plasma corticosterone levels that resemble moderate stress (de Quervain et al., 1998; Okuda et al., 2004).

Cannula implantation. Rats, adapted to the vivarium for at least 1 week, were anesthetized with sodium pentobarbital $(50 \mathrm{mg} / \mathrm{kg}$, i.p.), given atropine sulfate $(0.4 \mathrm{mg} / \mathrm{kg}$, i.p. $)$ to maintain respiration, and subsequently injected with $3.0 \mathrm{ml}$ of saline to facilitate clearance of these drugs and prevent dehydration. The skull was positioned in a stereotaxic frame (Kopf Instruments), and two stainless-steel guide cannulae (23 gauge; Small Parts) were implanted bilaterally with the cannula tips 2.0 $\mathrm{mm}$ above the insular cortex [ $15 \mathrm{~mm}$ long; coordinates: anteroposterior (AP), $+1.2 \mathrm{~mm}$ relative to bregma; mediolateral (ML), $\pm 5.5 \mathrm{~mm}$ from midline; dorsoventral (DV), $4.5 \mathrm{~mm}$ below skull surface] or $1.5 \mathrm{~mm}$ above the dorsal hippocampus (11 mm long; AP, $-3.4 \mathrm{~mm}$; ML, \pm 1.7 $\mathrm{mm} ; \mathrm{DV},-2.7 \mathrm{~mm})$. The coordinates were based on the atlas of Paxinos and Watson (2005). The cannulae were affixed to the skull with two anchoring screws and dental cement. Stylets (11- or 15-mm-long 00insect dissection pins), inserted into each cannula to maintain patency, were removed only for the infusion of drugs. After surgery, the rats were retained in an incubator until recovered from anesthesia and were then returned to their home cages. The rats were allowed to recover for a minimum of $10 \mathrm{~d}$ before initiation of training.

Local drug infusions. The HDAC inhibitor sodium butyrate (NaBut; 1 or $10 \mu \mathrm{g}$; Millipore) was dissolved in saline and infused into the insular cortex or hippocampus immediately after object training. For the second experiment, NaBut $(1$ or $10 \mu \mathrm{g})$ or corticosterone conjugated to a membrane-impermeable bovine serum albumin (BSA) molecule (cort: BSA; 3 or 10 ng; Sigma-Aldrich) was infused into the insular cortex after object training either alone or together with the specific GR antagonist RU 38486 (10 ng; Roussel UCLAF), the specific mineralocorticoid receptor (MR) antagonist RU 28318 (10 ng; Roussel UCLAF), or the selective and cell-permeable cAMP-dependent protein kinase (PKA) inhibitor PKI 14-22 amide (0.3 $\mu \mathrm{g}$; Calbiochem). For this second experiment, $\mathrm{NaBut}$ and the antagonists were dissolved in a vehicle containing $0.5 \%$ ethanol in saline, whereas the cort:BSA drug solutions were made in a vehicle containing $0.5 \%$ ethanol and $0.1 \%$ BSA in saline. The use of BSA-conjugated steroid hormones is a standard approach in steroid biology to demonstrate specific membrane effects (Zheng et al., 1996). For bilateral infusions into the insular cortex, the injection needles protruded 
$2.0 \mathrm{~mm}$ beyond the cannula tip, and a $0.5 \mu \mathrm{l}$ injection volume of drug or vehicle per hemisphere was infused over a period of $35 \mathrm{~s}$. For bilateral infusions into the hippocampus, the injection needles protruded $1.5 \mathrm{~mm}$ beyond the cannula tip, and a $0.5 \mu$ injection volume of drug or vehicle per hemisphere was infused over a period of $35 \mathrm{~s}$. The injection needles were retained within the cannulae for an additional $20 \mathrm{~s}$ after drug infusion to maximize diffusion.

Cannula placement verification. The rats were anesthetized deeply with sodium pentobarbital (100 mg/kg, i.p.) and perfused transcardially with $0.9 \%$ saline followed by $4 \%$ formaldehyde. After decapitation, the brains were removed and immersed in fresh $4 \%$ formaldehyde. At least $24 \mathrm{~h}$ before sectioning, the brains were transferred to a $30 \%$ sucrose solution for cryoprotection. Coronal sections of $50 \mu \mathrm{m}$ were cut on a freezing microtome, mounted on gelatin-coated slides, stained with cresyl violet, and examined by light microscopy. Rats with injection needle placements outside the insular cortex or the hippocampus or with extensive tissue damage at the injection needle site were excluded from analyses.

Histone prep. Rats were deeply anesthetized with pentobarbital $1 \mathrm{~h}$ after training and drug treatment, and brains were rapidly removed and flash frozen by submersion for $2 \mathrm{~min}$ in a beaker filled with 2-methylbutane sitting in a dry-ice ethanol bath. Hippocampal and insular cortex tissue was dissected from frozen $1 \mathrm{~mm}$ coronal slices using a $1 \mathrm{~mm}$ brain punch (Stoelting). Six punches were collected per $1 \mathrm{~mm}$ slice in hippocampus (AP, -2.8 to $-3.8 \mathrm{~mm}$; ML, $\pm 1.0-4.0 \mathrm{~mm}$; DV, 2.5$3.5 \mathrm{~mm}$ ). Two punches were collected per $1 \mathrm{~mm}$ slice (three slices total collected) in insular cortex (approximate range of coordinates encompassing three slices: $\mathrm{AP},+2.7$ to $-0.3 \mathrm{~mm}$; ML, $\pm 4.0-6.0 \mathrm{~mm}$; DV, $5.0-7.0 \mathrm{~mm}$ ). Twelve total punches from two rats were pooled to isolate histone proteins for both hippocampus and insular cortex. Tissue was homogenized in a hypotonic lysis buffer $(10 \mathrm{~mm}$ Tris, $\mathrm{pH} 8.0,1 \mathrm{~mm}$ EDTA, $2.5 \mathrm{~mm}$ sodium pyrophosphate, $1 \mathrm{~mm}$ sodium orthovanadate, 1 mM PMSF, and 1\% Halt protease cocktail inhibitor) in $1.5 \mathrm{ml}$ Eppendorf tubes using a $200 \mu \mathrm{l}$ pipettor. Lysates were then centrifuged at 16.1 relative centrifugal force (RCF) for $15 \mathrm{~min}$ at $4^{\circ} \mathrm{C}$. The supernatant was collected, leaving the nuclear pellet for acid extraction $(0.2 \mathrm{M} \mathrm{HCl} / 10 \%$ glycerol). The nuclear pellet and acid buffer were placed on ice for $5 \mathrm{~min}$ and then centrifuged at $16.1 \mathrm{RCF}$ for $30 \mathrm{~min}$ at $4^{\circ} \mathrm{C}$. Next, the acid supernatant was collected and ice-cold acetone was added to the pellet and placed on ice for $5 \mathrm{~min}$ before being centrifuged at 16.1 RCF for 30 $\min$ at $4^{\circ} \mathrm{C}$. Finally, the supernatant was removed and remaining histone pellet was placed at room temperature (RT) for $5 \mathrm{~min}$ (for remaining acetone to evaporate) before being resuspended in $9 \mathrm{~m}$ urea.

Western blot analysis. Final protein concentration of histone samples was determined using the Bio-Rad protein assay and bovine serum albumin (BSA) standards. Tissue samples and control histones (+/- NaBut; Millipore) were prepared in a standard $5 \times$ SDS/PAGE sample buffer ( $1 \mathrm{M}$ Tris, $\mathrm{pH} 6.8,20 \% \mathrm{v} / \mathrm{v}$ glycerol, $10 \% \mathrm{w} / \mathrm{v}$ SDS, $0.05 \%$ bromophenol blue, and $10 \mathrm{~mm} 2-\beta$-mercapto-ethanol). Thirty micrograms of protein were loaded per well and run at $40 \mathrm{~mA}$ for $2 \mathrm{~h}$ on a $5 \%$ stacking polyacrylamide gel and then at $50 \mathrm{~mA}$ for $5 \mathrm{~h}$ on a $12.5 \%$ resolving gel. Electrophoretic transfer was then performed over $5 \mathrm{~h}$ at $500 \mathrm{~mA}$ onto a polyvinylidene difluoride (Whatman Westran Clear Signal) membrane. Membranes were blocked for $2 \mathrm{~h}$ at RT in blocking solution [5\% nonfat milk/Trisbuffered saline with Tween 20 (TBS-T)] and then incubated in primary antibodies (1:3000 anti-acetylated histone H3, Millipore; 1:1000 rabbit anti-actin, Millipore) with agitation overnight at $4^{\circ} \mathrm{C}$. Membranes were then rinsed three times quickly in TBS-T and then three additional times ( 5 min each) with agitation, also in TBS-T. Next, membranes were incubated for $1 \mathrm{~h}$ at RT in a 1:5000 dilution of polyclonal anti-goat rabbit HRP secondary antibody (Millipore) or 1:25,000 dilution of anti-mouse Ig HRP antibody (GE Healthcare) in blocking solution. Membranes were then rinsed three times quickly in TBS and then three additional times (5 min each) with agitation, also in TBS. For chemiluminescent detection, the ECL Plus Western Blotting Detection System (GE Healthcare) was used according to the manufacturer's instructions and detected using the Typhoon Trio ${ }^{+}$and analyzed using ImageQuant (GE Healthcare) software.

Immunohistochemistry. One hour after object training and drug infusion into the insular cortex, rats were anesthetized deeply with sodium pentobarbital (100 mg/kg, i.p.) and perfused transcardially with ice-cold $0.1 \mathrm{M}$ PBS, pH 7.4, followed by ice-cold $4 \%$ paraformaldehyde in PBS, pH 7.4. The brains were removed, postfixed overnight at $4^{\circ} \mathrm{C}$, and then transferred to $30 \%$ sucrose for $48 \mathrm{~h}$ at $4^{\circ} \mathrm{C}$. Frozen coronal sections were cut at a thickness of $20 \mu \mathrm{m}$ on a cryostat and collected in $0.1 \mathrm{M}$ PBS at the level corresponding to the insular cortex (0.2-2.2 $\mathrm{mm}$ anterior to bregma).

Floating sections were rinsed in $0.1 \%$ Triton X-100 (Fisher) in PBS, rinsed in PBS, and then blocked for $1 \mathrm{~h}$ at RT in $8 \%$ normal goat serum (NGS, Jackson ImmunoResearch Laboratories) with $0.3 \%$ Triton X-100 in PBS. Sections were rinsed in PBS and incubated overnight at $4^{\circ} \mathrm{C}$ in $2 \%$ NGS, $0.3 \%$ Triton X-100 in PBS with primary antibody (acetylHistone-H3 primary antibody, 1:1000, Millipore; or phospho-CREB primary antibody, 1:25, Cell Signaling). The sections were then rinsed in PBS and incubated for $2 \mathrm{~h}$ at RT with goat anti-rabbit IgG-FITC secondary antibody (1:1000, Millipore Bioscience Research Reagents). Sections were rinsed again in PBS and mounted on slides using ProLong Gold antifade reagent with DAPI (Invitrogen). Twelve-bit grayscale images of the insular cortex were acquired with a BX51TF-5 Olympus Microscope using a $\times 20$ objective and digitized using a Retiga-2000RV camera. A single optimized acquisition exposure time was used for all images acquired from a particular slide.

Statistics. Data were expressed as mean \pm SEM. Statistical analysis used unpaired $t$ tests to compare two groups or one- or two-way ANOVAs for multiple comparisons, when appropriate, followed by post hoc comparison tests. One-sample $t$ tests were used to determine whether the discrimination index was different from zero and thus whether learning had occurred. A probability level of $<0.05$ was accepted as statistical significance for all tests.

\section{Results}

\section{Posttraining corticosterone enhances memory of different components of object training}

This experiment investigated whether corticosterone administered systemically to rats immediately after object training enhances long-term memory of different components of the training experience. All rats were subjected to a 3 min training trial during which they could freely explore two identical objects placed in the corners of the apparatus, followed immediately by a systemic injection of either corticosterone ( $1 \mathrm{mg} / \mathrm{kg}$, s.c.) or vehicle. This dose of corticosterone is known to induce plasma corticosterone levels that resemble moderate stress (de Quervain et al., 1998; Okuda et al., 2004). To determine whether animals exhibit a long-term memory for the object seen during the training trial (referred to as ORM), rats were given a $24 \mathrm{~h}$ retention test in which one object was familiar and the other object was novel. If the animal generates a long-term memory for the familiar object, it will spend significantly more time exploring the novel object during the retention test. To determine whether animals exhibit a long-term memory for the location of an object (referred to as OLM), other rats were given a $24 \mathrm{~h}$ retention test in which both objects were familiar, yet one was placed in a novel location. If the animal generates a long-term memory for the association between an object and its location, it will spend significantly more time exploring the object in a novel location. A diagram of the experimental design is shown in Figure $1 a$.

\section{ORM test}

Corticosterone treatment immediately after the training trial enhanced long-term memory for the familiar object. The vehicle controls did not express significant $24 \mathrm{~h}$ retention of the familiar object (one-sample $t$ test: $t_{(7)}=1.33, p=0.23$ ), but immediate posttraining administration of corticosterone enhanced $24 \mathrm{~h}$ retention performance ( $p=0.02$, as compared to vehicle) (Fig. 1b). Also, a one-sample $t$ test indicated that rats treated with corticosterone exhibited a significant exploration preference for the novel object $\left(t_{(7)}=4.12 ; p=0.005\right)$. The vehicle and corticoste- 
a

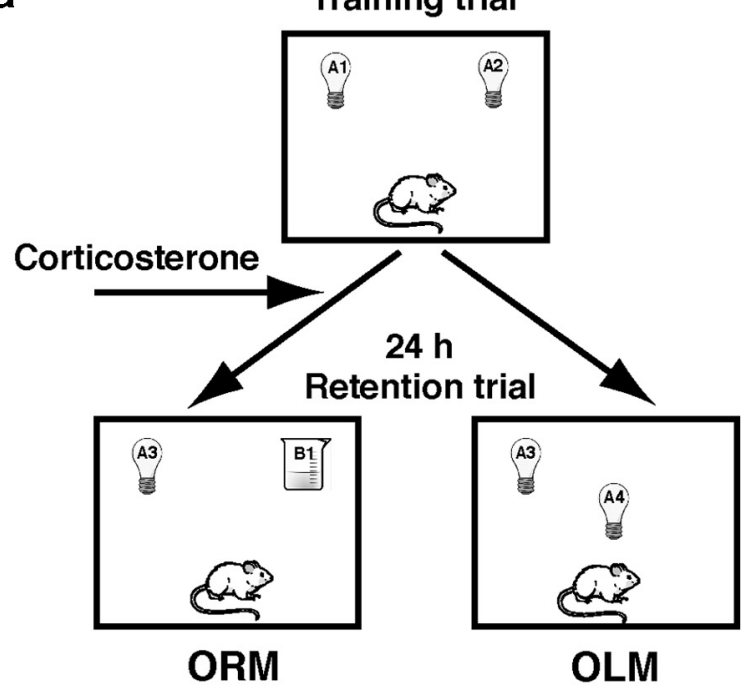

b

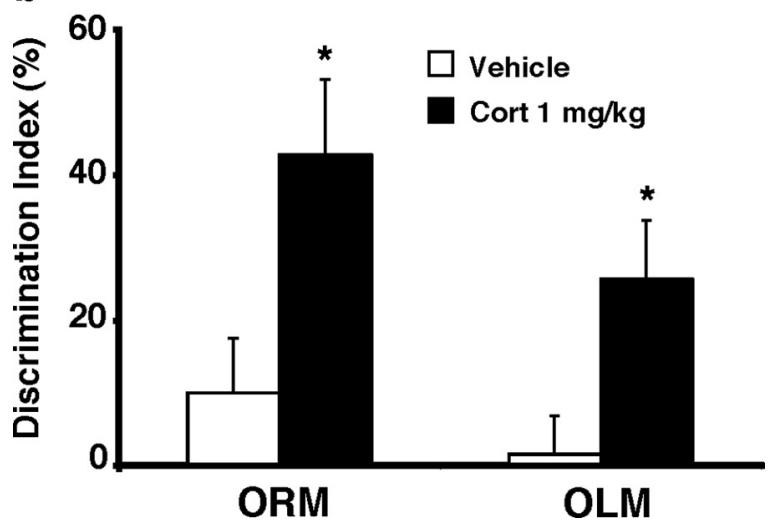

Figure 1. Systemic posttraining corticosterone enhances the retention of object recognition and object location memory. $\boldsymbol{a}$, On the $3 \mathrm{~min}$ training trial, rats were allowed to freely explore two identical objects placed in the corners of a novel environment followed immediately by a systemic injection of either vehicle or corticosterone $(1 \mathrm{mg} / \mathrm{kg}$, s.c.). Retention of the training was tested $24 \mathrm{~h}$ later by placing the rat in the same apparatus either with one object replaced by a novel object placed in the same location as objects during training (ORM) or by moving one familiar object to a novel location (OLM). $\boldsymbol{b}$, Systemic injection of corticosterone (1 mg/kg, s.c.) enhances $24 \mathrm{~h}$ retention of both ORM and OLM. Data represent discrimination index (percentage), expressed as mean \pm SEM. ${ }^{*} p<0.05$ compared with the corresponding vehicle control group ( $n=8-11$ per group).

rone groups did not differ in total exploration time of the two objects during either the training or retention test (data not shown).

\section{OLM test}

Corticosterone treatment immediately after the training trial also enhanced long-term memory for the location of the object. The vehicle controls did not express significant $24 \mathrm{~h}$ retention of the location of the familiar object (one-sample $t$ test: $t_{(9)}=0.29, p=$ 0.78 ) (Fig. 1b). However, corticosterone treatment enhanced $24 \mathrm{~h}$ retention performance ( $p=0.02$, as compared to vehicle). Also, a one-sample $t$ test indicated that rats treated with corticosterone exhibited a significant exploration preference for the object placed in the novel location $\left(t_{(10)}=3.15 ; p=0.01\right)$. The vehicle and corticosterone groups did not differ in total exploration time of the two objects during either the training or retention test (data not shown).

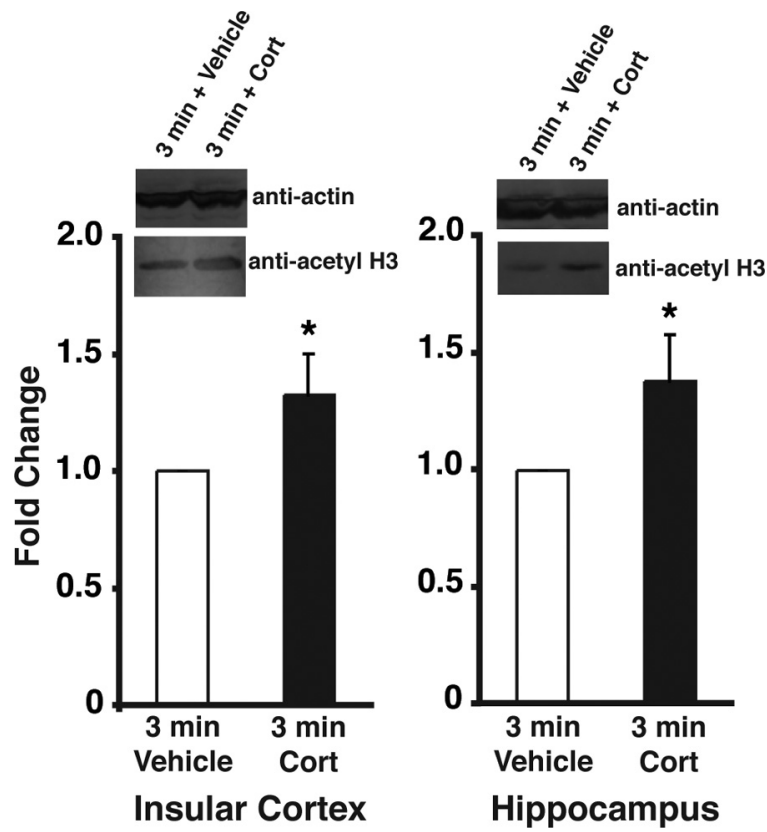

Figure 2. Systemic corticosterone increases acetylation of histone $\mathrm{H} 3$ at Lys-14 in the insular cortex and hippocampus. Rats received training identical to that in the experiment shown in Figure $1 a$ followed immediately by a systemic injection of either vehicle or corticosterone (1 mg/kg, s.c.). One hour following training, punches from the insular cortex and hippocampus were taken to isolate histone proteins that were then separated by SDS-PAGE. In the left panel, Western blot analysis using anti-acetyl lysine 14 histone $\mathrm{H} 3$ antibodies shows an increase in acetylation following corticosterone treatment as compared to vehicle in the insular cortex. Similarly, in the right panel, Western blot analysis using anti-acetyl lysine 14 histone $\mathrm{H} 3$ antibodies shows an increase in acetylation following corticosterone treatment as compared to vehicle in the hippocampus. ${ }^{*} p<0.05$ compared with the corresponding vehicle control group ( $n=9-11$ per group).

Posttraining corticosterone increases histone $\mathrm{H} 3$, lysine 14 acetylation in the insular cortex and hippocampus

Findings of several studies indicate that corticosterone acts in different brain regions to enhance the consolidation of memory for different training experiences (Roozendaal et al., 2008). As findings indicate that object recognition memory involves the insular cortex (Bermudez-Rattoni et al., 2005), whereas object location memory involves the hippocampus (Balderas et al., 2008), systemically administered corticosterone might act in the insular cortex and hippocampus to enhance the consolidation of object recognition and object location memory, respectively. To determine whether corticosterone administered immediately after object training altered histone acetylation in these two brain regions, we used Western blot analysis to examine histone $\mathrm{H} 3$, lysine 14 acetylation (H3K14ac) of histone preps. Rats were subjected to the same training procedure described in Figure 1, followed immediately by an injection of corticosterone $(1 \mathrm{mg} / \mathrm{kg}$, s.c.) or vehicle. One hour later, tissue was collected and acidextracted histones were prepared from insular cortex or hippocampal nuclear lysates and separated by SDS-PAGE. As shown in Figure 2, corticosterone increased histone acetylation levels in both the insular cortex ( $n=9$ for both corticosterone and vehicle; $p=0.04)$ and the hippocampus $(n=11$ for both corticosterone and vehicle; $p=0.03$ ), as compared to vehicle-treated animals.

Double dissociation between HDAC inhibition in the insular cortex and hippocampus on memory of object recognition and object location

Thus far, we found that corticosterone administered immediately after object training significantly enhanced the consolidation of 


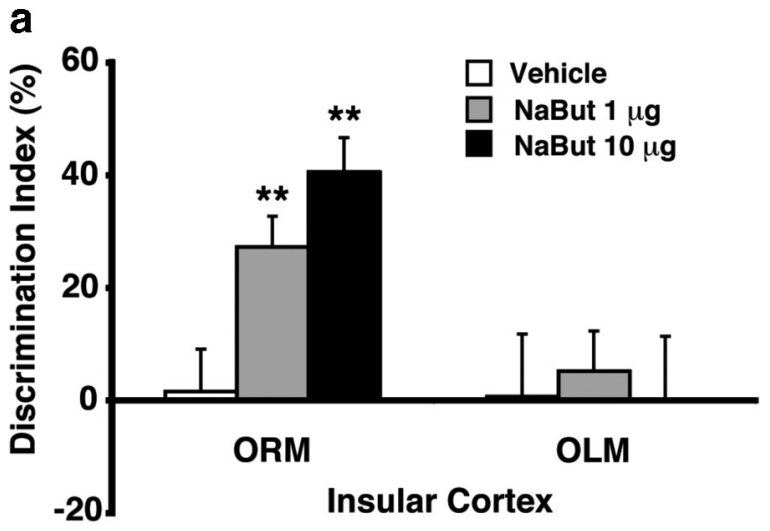

b

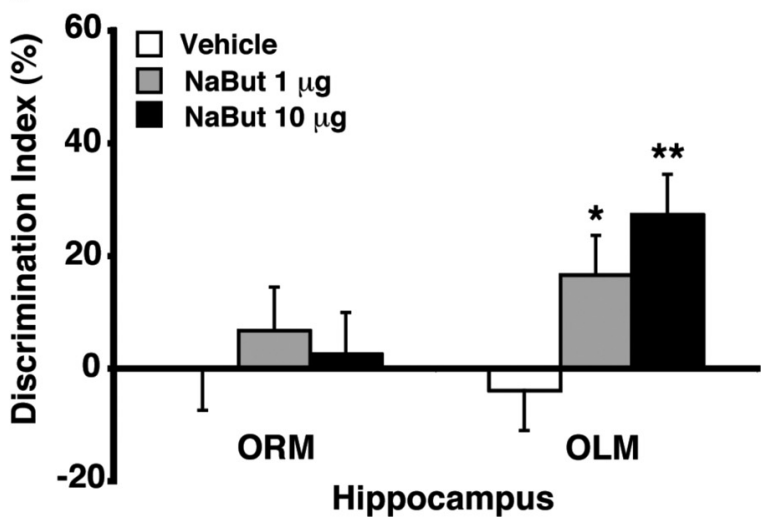

Figure 3. Double dissociation between posttraining HDAC inhibitor infusion into the insular cortex and hippocampus on the consolidation of object recognition and object location memory. $\boldsymbol{a}$, The HDAC inhibitor NaBut (1 or $10 \mu \mathrm{g}$ in $0.5 \mu \mathrm{l}$ ) infused into the insular cortex immediately after training enhanced $24 \mathrm{~h}$ retention of ORM, but not OLM. $\boldsymbol{b}$, The HDAC inhibitor NaBut (1 or $10 \mu \mathrm{g}$ in $0.5 \mu \mathrm{l}$ ) infused into the hippocampus immediately after training enhanced $24 \mathrm{~h}$ retention of OLM, but not ORM. Data represent discrimination index (percentage), expressed as mean \pm SEM. ${ }^{*} p<0.05 ;{ }^{* *} p<0.01$ compared with the corresponding vehicle control group ( $n=7-12$ per group).

object recognition and object location memory as well as elevated acetylated histone levels in both the insular cortex and hippocampus. To determine whether the elevated histone acetylation levels in these two brain regions play a role in the memory-enhancing effect of corticosterone, we examined the effect of increasing histone acetylation in each of these two brain regions with immediate posttraining infusions of the HDAC inhibitor NaBut on $24 \mathrm{~h}$ retention of object recognition and object location memory.

\section{Insular cortex}

NaBut $(1$ or $10 \mu \mathrm{g}$ in $0.5 \mu \mathrm{l}$ ) infused into the insular cortex immediately after object training significantly enhanced $24 \mathrm{~h}$ retention of object recognition memory $\left(F_{(2,29)}=16.03 ; p<\right.$ 0.0001 ) (Fig. $3 a$ ). One-sample $t$ tests revealed that rats administered either dose of $\mathrm{NaBut}$ exhibited a significance preference for the novel object $\left(1 \mu \mathrm{g}: t_{(11)}=5.03 ; p=0.0004 ; 10 \mu \mathrm{g}: t_{(9)}=6.45\right.$; $p<0.0001)$. In contrast, NaBut infused into the insular cortex did not enhance retention of object location memory $\left(F_{(2,25)}=0.08 ; p=\right.$ $0.92)$. The different groups did not differ in total exploration time of the two objects during either the training or retention test (data not shown).

\section{Hippocampus}

Posttraining infusions of NaBut ( 1 or $10 \mu \mathrm{g}$ in $0.5 \mu \mathrm{l}$ ) into the dorsal hippocampus did not enhance $24 \mathrm{~h}$ retention of object recognition memory $\left(F_{(2,20)}=0.26 ; p=0.78\right)$, but enhanced the retention of object location memory $\left(F_{(2,23)}=4.66 ; p=0.02\right)$ (Fig. $3 b$ ). One-sample $t$ tests indicated that rats administered either dose of NaBut exhibited a significance preference for the object placed in the novel location $\left(1 \mu \mathrm{g}: t_{(7)}=2.37 ; p=0.05 ; 10\right.$ $\left.\mu \mathrm{g}: t_{(9)}=3.82 ; p=0.004\right)$. The different groups did not differ in total exploration time of the two objects during either the training or retention test (data not shown).

Corticosterone administration into the insular cortex enhances object recognition memory and increases phospho-CREB levels via an activation of membrane-associated GRs

Extensive evidence indicates that glucocorticoids act in the hippocampus to enhance spatial and/or contextual memory (Roozendaal et al., 2008). However, considerably less is known concerning direct actions of glucocorticoids in the insular cortex (Miranda et al., 2008). Although this brain region has traditionally been associated with subserving memory for taste and disgust, recent findings suggest that it may play a much more general role in recognition memory (Bermúdez-Rattoni, 2004; Reed et al., 2004; Bermudez-Rattoni et al., 2005). This experiment investigated whether glucocorticoids act in the insular cortex to enhance the consolidation of object recognition memory and whether the effect involves PKA signaling to activate phospho-CREB. Although the classic mechanism of glucocorticoid-induced memory enhancement is considered to involve intracellular receptors, which translocate to the nucleus and act as a transcription factor (Beato and Sánchez-Pacheco, 1996), we previously reported that the rapid effects of glucocorticoids on the cAMP/PKA pathway likely involve the activation of a membrane-associated receptor (Roozendaal et al., 2008). Therefore, this experiment examined whether immediate posttraining infusions of corticosterone conjugated to a membrane-impermeable bovine serum albumin molecule (cort:BSA) administered into the insular cortex after object training enhance $24 \mathrm{~h}$ retention of object recognition memory and whether this memory enhancement depends on an activation of the PKA signaling cascade.

As is shown in Figure $4 a$, immediate posttraining infusions of cort:BSA (3 or $10 \mathrm{ng}$ in $0.5 \mu \mathrm{l}$ ) administered into the insular cortex enhanced $24 \mathrm{~h}$ retention of object recognition memory $\left(F_{(2,27)}=6.68 ; p=0.004\right)$. One-sample $t$ tests indicated that rats given either dose of cort:BSA showed a significant preference for the novel object ( 3 ng: $t_{(8)}=2.66 ; p=0.03 ; 10$ ng: $t_{(10)}=5.90 ; p=$ $0.0002)$. This cort:BSA-induced memory enhancement likely involves an activation of (membrane-bound) GRs as coadministration of the GR antagonist RU 38486 (10 ng) blocked the cort: BSA-induced retention enhancement. Two-way ANOVA for discrimination index revealed a significant cort:BSA effect $\left(F_{(2,56)}=3.30, p=0.04\right)$, a significant RU 38486 effect $\left(F_{(1,56)}=\right.$ $15.91, p=0.0002)$, and a significant interaction between both factors $\left(F_{(2,56)}=3.44, p=0.04\right)$. In contrast, the MR antagonist RU 28318 (10 ng) infused into the insular cortex immediately posttraining did not block the cort:BSA-induced retention enhancement. Two-way ANOVA for discrimination index revealed a significant cort:BSA effect $\left(F_{(2,54)}=22.75, p<0.0001\right)$, but no significant RU 28318 effect $\left(F_{(1,54)}=0.41, p=0.53\right)$ or interaction between both factors $\left(F_{(2,54)}=0.60, p=0.55\right)$.

In support of the view that cort:BSA enhances memory consolidation of object training via an activation of the PKA signaling pathway, coadministration of the selective PKA inhibitor PKI $14-22$ amide $(0.3 \mu \mathrm{g})$ blocked the cort:BSA-induced retention 


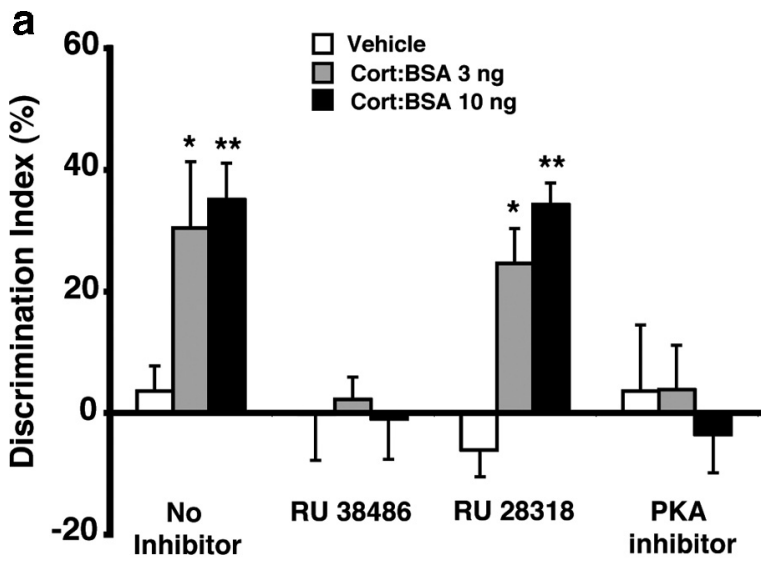

b

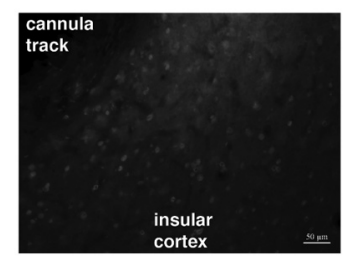

Vehicle anti-pCREB antibody

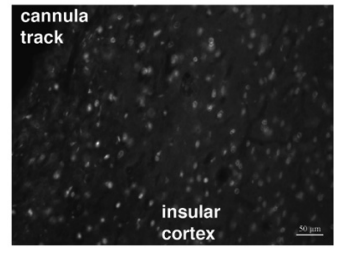

Cort:BSA anti-pCREB antibody

Figure 4. Posttraining infusions of the membrane-impermeable glucocorticoid cort:BSA into the insular cortex enhances retention of object recognition memory. $\boldsymbol{a}$, Immediate posttraining infusions of cort:BSA ( 3 or $10 \mathrm{ng}$ in $0.5 \mu \mathrm{l}$ ) into the insular cortex enhanced $24 \mathrm{~h}$ retention of ORM. This retention enhancement was blocked by coinfusion of the selective glucocorticoid receptor antagonist RU 38486 (10 ng) or protein kinase A inhibitor PKI 14-22 amide $(0.3 \mu \mathrm{g})$, but not the mineralocorticoid receptor antagonist RU $28318(10 \mathrm{ng})$. Data represent discrimination index (percentage), expressed as mean \pm SEM. ${ }^{*} p<0.05 ;{ }^{* *} p<0.01$ compared with the corresponding vehicle control group ( $n=10-11$ per group). $\boldsymbol{b}$, Immediate posttraining infusion of cort:BSA (10 ng) into the insular cortex increases the expression of phosphorylated cAMP response-element binding (phospho-CREB) protein around the infusion site $1 \mathrm{~h}$ after training.

enhancement. Two-way ANOVA for discrimination index revealed a nonsignificant cort:BSA effect $\left(F_{(2,54)}=1.54, p=0.22\right)$, a significant PKI effect $\left(F_{(1,54)}=10.16, p=0.002\right)$, and a significant interaction between both factors $\left(F_{(2,54)}=3.53, p=0.04\right)$ (Fig. 4a).

To determine further whether cort:BSA influences the PKA signaling pathway, we examined phospho-CREB immunoreactivity in the insular cortex near the cannula termination site in perfusion-fixed coronal brain slices. Animals were killed $1 \mathrm{~h}$ after training and drug infusion. As is shown in Figure $4 b$, vehicletreated rats did not show any detectable phospho-CREB immunoreactivity in the insular cortex. However, cort:BSA (10 ng in $0.5 \mu \mathrm{l}$ ) treatment resulted in a clear phosphorylation of CREB. Nuclear staining with DAPI demonstrated that there was no observable damage to the insular cortex beyond the injection site due to the delivery of vehicle or cort:BSA (data not shown).

Insular cortex infusions of a GR antagonist or PKA inhibitor, but not an MR antagonist, block NaBut-induced retention enhancement of object recognition memory

In a previous study we demonstrated that inducing a histone hyperacetylated state via HDAC inhibition is not sufficient to enhance hippocampal synaptic plasticity (Vecsey et al., 2007), but that the effect depends on a phospho-CREB:CBP interaction, presumably to recruit the basal transcription machinery for gene expression. As the above-mentioned findings indicate that glu-
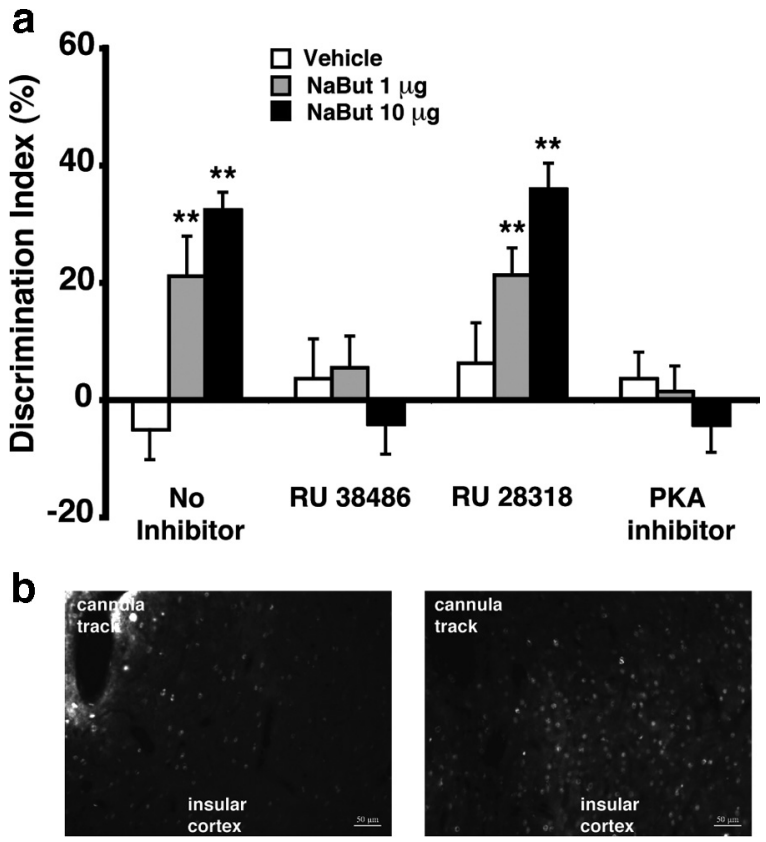

Vehicle anti-histone H3 lys14

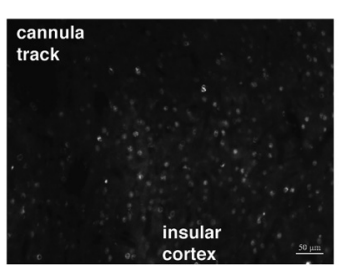

NaBut anti-histone H3 lys14

Figure 5. Glucocorticoid activity is required to enable the memory-enhancing effect of HDAC inhibitor infusion into the insular cortex on object recognition. $\boldsymbol{a}$, Immediate posttraining infusions of the HDAC inhibitor NaBut ( 1 or $10 \mu \mathrm{g}$ in $0.5 \mu \mathrm{l}$ ) into the insular cortex enhanced $24 \mathrm{~h}$ retention of $O R M$. This retention enhancement was blocked by coinfusion of the selective glucocorticoid receptor antagonist RU 38486 (10 ng) or protein kinase A inhibitor PKI 14-22 amide $(0.3 \mu \mathrm{g})$, but not the mineralocorticoid receptor antagonist RU 28318 (10 ng). Data represent discrimination index (percentage), expressed as mean \pm SEM. ${ }^{* *} p<0.01$ compared with the corresponding vehicle control group ( $n=9-11$ per group). $\boldsymbol{b}$, Immediate posttraining infusion of NaBut ( 1 or $10 \mu \mathrm{g}$ in $0.5 \mu \mathrm{l}$ ) into the insular cortex increased acetylation of histone $\mathrm{H} 3$ at lysine 14 around the infusion site $1 \mathrm{~h}$ after training.

cocorticoids increase histone acetylation as well as phosphoCREB levels in the insular cortex, we determined next whether the memory enhancement induced by NaBut infusions into the insular cortex requires concurrent glucocorticoid signaling. This experiment examined whether immediately posttraining bilateral infusions of a GR or MR antagonist or PKA inhibitor into the insular cortex would block $24 \mathrm{~h}$ retention enhancement of object recognition memory induced by concurrently administered NaBut.

As is shown in Figure $5 a$, NaBut ( 1 or $10 \mu \mathrm{g}$ in $0.5 \mu \mathrm{l}$ ) infusions into the insular cortex after object training enhanced $24 \mathrm{~h}$ retention of object recognition memory $(1 \mu \mathrm{g}: p=0.001 ; 10 \mu \mathrm{g}$ : $p=0.0001)$. The GR antagonist RU 38486 (10 ng) infused into the insular cortex immediately after the training trial blocked the retention enhancement induced by NaBut. Two-way ANOVA for discrimination index revealed a significant NaBut effect $\left(F_{(2,53)}=4.64, p=0.01\right)$, a significant RU 38486 effect $\left(F_{(1,53)}=\right.$ 10.37, $p=0.002)$, and a significant interaction between both factors $\left(F_{(2,53)}=8.65, p=0.0008\right)$. In contrast, the MR antagonist RU 28318 infused into the insular cortex immediately after object training did not block the NaBut-induced retention enhancement. Two-way ANOVA for discrimination index revealed a significant NaBut effect $\left(F_{(2,56)}=20.42, p<0.0001\right)$, but no significant RU 28318 effect $\left(F_{(1,56)}=1.33, p=0.25\right)$ or interaction between both factors $\left(F_{(2,56)}=0.57, p=0.57\right)$. The pattern of results for the PKA inhibitor PKI infused into the insular cortex together with NaBut was similar to that of the GR antagonist. Two-way ANOVA for discrimination index revealed a significant 
NaBut effect $\left(F_{(2,55)}=5.32, p=0.008\right)$, a significant PKI effect $\left(F_{(1,55)}=15.83, p=0.0002\right)$, and a significant interaction between both factors $\left(F_{(2,55)}=11.39, p<0.0001\right)$. Thus, these findings indicate that the memory-enhancing effect induced by NaBut infusions requires a coactivation of GRs and PKA, but not MRs.

To determine the effect of NaBut infusions on histone acetylation in the insular cortex, we examined the level of lysine 14 acetylation on histone $\mathrm{H} 3$ ( $\mathrm{H} 3 \mathrm{~K} 14 \mathrm{ac}$ ) near the cannula termination site in perfusion-fixed coronal brain slices using immunohistochemistry. As is shown in Figure $5 b$, NaBut ( $10 \mu \mathrm{g}$ in $0.5 \mu \mathrm{l}$ ) treatment resulted in markedly increased histone $\mathrm{H} 3$ acetylation, as compared to vehicle-treated rats. Nuclear staining with DAPI demonstrated that there was no observable damage to the insular cortex beyond the injection site due to the delivery of vehicle or $\mathrm{NaBut}$ (data not shown). This finding demonstrates that $\mathrm{NaBut}$ increased histone acetylation as predicted.

Posttraining corticosterone injections enhance retention of object location memory, but not object recognition memory, in a CREB:CBP-dependent manner

To examine whether CBP is involved in the mechanisms underlying corticosterone-dependent enhancement of memory, we used genetically modified $C b p$ mutant mice carrying a triple point mutation in the phospho-CREB (KIX) binding domain of CBP (CBP ${ }^{\mathrm{KIX} / \mathrm{KIX}}$ mice) (Kasper et al., 2002). In a previous study we used these Cbp mutant mice to demonstrate that HDAC inhibition, by either NaBut or trichostatin A, enhances synaptic plasticity in the hippocampus in a CREB:CBP-dependent manner (Vecsey et al., 2007).

We first examined whether corticosterone enhances $24 \mathrm{~h}$ retention of object recognition and object location memory in wild-type mice. Mice were subjected to a 3 min training period, which does not lead to long-term memory (Stefanko et al., 2009). Immediately after training, mice were administered either corticosterone ( $1 \mathrm{mg} / \mathrm{kg}$, s.c.) or vehicle and given a retention test $24 \mathrm{~h}$ later. As shown in Figure $6 a$, corticosterone-treated mice $(n=$ 11) exhibited significantly enhanced memory for the familiar object compared with vehicle controls $(n=10 ; p=0.03)$. Similar effects of corticosterone were observed on object location memory. Corticosterone-treated mice $(n=8)$ exhibited significantly enhanced retention for the location of the familiar object as compared to vehicle controls ( $n=8 ; p=0.005$ ) (Fig. $6 a$, right). Thus, comparable to the effect of corticosterone injections in rats (Fig. 1 ), these findings indicate that corticosterone enhances memory of object recognition and object location. Posttraining corticosterone transforms what is learned by just a 3 min training period (that does not normally lead to long-term memory by itself) into an event that does result in long-term memory.

We next examined the role of CBP in corticosteronedependent long-term memory formation in $\mathrm{CBP}^{\mathrm{KIX} / \mathrm{KIX}}$ homozygous knock-in mice (Kasper et al., 2002). We previously found these mice to exhibit normal short-term memory for object recognition, but impaired long-term memory (Wood et al., 2006; Stefanko et al., 2009). Cbp ${ }^{\mathrm{KIX} / \mathrm{KIX}}$ homozygous mice and wild-type $C b p^{+/+}$littermates were subjected to a 10 min training trial (which leads to long-term memory formation in wild-type mice; Stefanko et al., 2009), and then immediately afterward given an injection of either corticosterone ( $1 \mathrm{mg} / \mathrm{kg}$, s.c.) or vehicle. $C b p^{\mathrm{KIX} / \mathrm{KIX}}$ homozygous mice treated with vehicle $(n=13)$ exhibited significantly impaired long-term memory compared with wild-type controls $(n=9, p=0.01)$. However, $C b p^{\mathrm{KIX} / \mathrm{KIX}}$ homozygous mice administered corticosterone $(n=11)$ exhib-
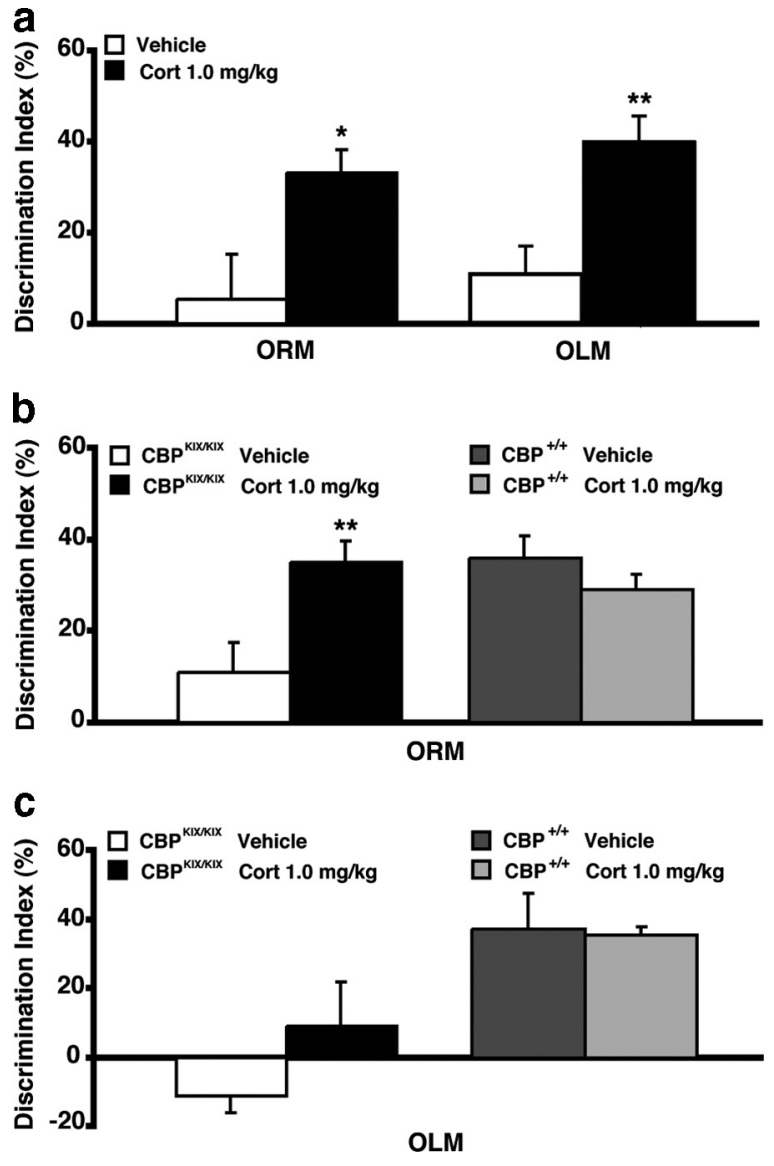

Figure 6. Systemic posttraining corticosterone ameliorates the long-term memory impairment for object recognition, but not object location, in CBP ${ }^{\mathrm{KIX} / \mathrm{KIX}}$ mice. $a$, Systemic injection of corticosterone ( $1 \mathrm{mg} / \mathrm{kg}$ ) enhances $24 \mathrm{~h}$ retention of ORM (left) as well as OLM (right) in C $57 \mathrm{BL} / 6$ mice ( $n=8-11$ per group). $\boldsymbol{b}$, CBP $^{\mathrm{KIX} / \mathrm{KIX}}$ mice that received 10 min of training immediately followed by an injection of corticosterone ( $1 \mathrm{mg} / \mathrm{kg}$, s.c.) displayed a significant preference for the novel object compared with vehicle-treated $\mathrm{CBP}^{\mathrm{KIX} / \mathrm{KIX}}$ mice in a $24 \mathrm{~h} 0 \mathrm{RM}$ retention test (left). Wild-type $\mathrm{CBP}^{+/+}$mice that received 10 min of training immediately followed by an injection of corticosterone exhibited a significant preference for the novel object similar to wild-type mice treated with vehicle (right) ( $n=9-13$ per group). c, CBP ${ }^{\mathrm{KIX} / \mathrm{KIX}}$ mice that received 10 min of training immediately followed by an injection of corticosterone exhibited no long-term memory, similar to vehicle-treated $\mathrm{CBP}^{\mathrm{KIX} / \mathrm{KIX}}$ mice, in a $24 \mathrm{~h} 0 \mathrm{LM}$ retention test (left). In contrast, wild-type $\mathrm{CBP}^{+/+}$mice that received 10 min of training immediately followed by an injection of corticosterone exhibited a significant preference for the familiar object in a novel location, similar to wild-type mice treated with vehicle (right) ( $n=5-9$ per group). ${ }^{*} p<0.05 ;{ }^{* *} p<0.01$ compared with the corresponding vehicle control group.

ited a significant preference for the novel object during the $24 \mathrm{~h}$ retention test (treatment $\times$ genotype interaction: $F_{(1,39)}=8.47$, $p=0.006$; Bonferroni post hoc analysis: $p=0.01$ ) (Fig. $6 b$, left). Wild-type mice administered corticosterone $(n=10)$ exhibited a preference for the novel object similar to wild-type mice treated with vehicle (Fig. $6 b$, right). Together, these results indicate that corticosterone ameliorates the long-term memory impairment for object recognition in $C b p^{\mathrm{KIX} / \mathrm{KIX}}$ homozygous mice.

In contrast, corticosterone was unable to ameliorate the longterm memory impairment for object location in $C b p^{\text {KIX/KIX }}$ homozygous mice. $C b p^{\mathrm{KIX} / \mathrm{KIX}}$ homozygous mice and wild-type $C b p^{+1+}$ littermates were subjected to a 10 min training period, and then immediately given an injection of either corticosterone $(1 \mathrm{mg} / \mathrm{kg}, \mathrm{s.c}$.) or vehicle. Similar to the object recognition data shown in Figure $6 b, C b p^{\mathrm{KIX} / \mathrm{KIX}}$ homozygous mice treated with vehicle $(n=8)$ exhibited significantly impaired 24 h retention of object location memory as compared to wild-type controls $(n=9$, 
$p=0.001)$. However, and most importantly, corticosterone treatment did not enhance $24 \mathrm{~h}$ retention of object location memory in $C b p^{\mathrm{KIX} / \mathrm{KIX}}$ homozygous mice $\left(n=6\right.$, genotype: $F_{(1,24)}=$ 16.04, $p=0.005$ ) (Fig. $6 c$, left). Wild-type mice that received corticosterone $(n=5)$ exhibited a preference for the novel object similar to wild-type mice treated with vehicle (Fig. $6 c$, right). These findings suggest that corticosterone enhances memory for object location in a CREB:CBP-dependent manner.

\section{Discussion}

These experiments examined how glucocorticoid activity and chromatin modification may interact to modulate long-term memory of object recognition and object location. Our previous studies demonstrated that glucocorticoids (Okuda et al., 2004; Roozendaal et al., 2006) as well as CBP and histone acetylation (Wood et al., 2006; Stefanko et al., 2009) are involved in modulating object recognition long-term memory processes. Here we show that corticosterone increases histone acetylation during the consolidation phase and enhances long-term memory of object recognition and object location. Furthermore, HDAC inhibition, which has been shown to enhance synaptic plasticity and longterm memory, enhances memory in a GR-dependent manner. All together, our data suggest that activation of membraneassociated GRs enhances memory consolidation via chromatin modification. However, as described below, the findings suggest some intriguing differences between glucocorticoid effects on hippocampus-dependent and hippocampus-independent aspects of object recognition memory.

We found that stress-level corticosterone treatment administered systemically immediately after the object training increased histone acetylation in the hippocampus and insular cortex and that these increases correlated with enhanced memory for both object recognition and object location. To determine whether the increased histone acetylation played a role in the retention enhancement of object recognition and object location memory, we administered the HDAC inhibitor NaBut to increase histone acetylation site specifically into either the hippocampus or the insular cortex. The HDAC inhibition in the insular cortex enhanced object recognition memory, but not object location memory. Conversely, HDAC inhibition in the hippocampus enhanced object location memory, but not object recognition memory. This double dissociation is consistent with other evidence showing that the hippocampus and insular cortex are involved in processing different forms of memory (BermudezRattoni et al., 2005; Balderas et al., 2008; Piterkin et al., 2008).

To understand how glucocorticoid signaling may be integrated with histone acetylation and CBP activity to regulate gene expression required for long-term memory formation, we used a cort:BSA conjugate. This conjugate does not cross the cell membrane and thus selectively activates adrenal steroid receptors on the cell surface (Chiyo et al., 2003). One potential caveat with using a BSA conjugate is that proteolytic mechanisms may separate BSA from a steroid hormone. However, the use of BSAconjugated steroid hormones is a standard approach in steroid biology to demonstrate specific membrane effects (Zheng et al., 1996). Posttraining infusions of cort:BSA into the insular cortex enhanced $24 \mathrm{~h}$ retention of object recognition memory. Using selective inhibitors to determine which receptor type is necessary for corticosterone-dependent enhanced memory, we found that GR-mediated signaling is critical, but MR-mediated signaling is not. Further, PKA inhibition also blocked the ability of cort:BSA to enhance memory. Together, the findings suggest that corticosterone activates putative membrane-bound GRs (Johnson et al.,
2005), resulting in PKA-dependent increased CREB phosphorylation in the insular cortex. These findings are consistent with previous evidence indicating that a specific GR agonist interacts with arousal-induced noradrenergic activation to rapidly increase PKA signaling and phospho-CREB activity (Roozendaal et al., 2002, 2006; A. Barsegyan, S. M. MacKenzie, B. D. Kurose, J. L. McGaugh, B. Roozendaal, unpublished observation). Such interactions between glucocorticoids and the noradrenergic signaling cascade may underlie the selective influence of glucocorticoids on memory of emotionally arousing experiences (Roozendaal et al., 2008). Interestingly, whereas previous studies have focused mainly on the basolateral amygdala, the present findings indicate that such interactions may also exist in other brain regions.

To examine whether HDAC inhibition was sufficient to enhance object recognition memory, we again used GR and MR specific inhibitors. The effect of the HDAC inhibitor NaBut on memory enhancement when delivered to the insular cortex was completely abolished by blocking GR activity, but not by blocking MR activity. Additionally, a PKA inhibitor also blocked the ability of HDAC inhibition to enhance memory in the insular cortex. These findings suggest that inducing a histone hyperacetylated state via HDAC inhibition is not sufficient to enhance long-term memory. It is still necessary to have upstream signaling via GR and PKA activity. Presumably, these signaling events are triggering steps necessary to activate transcription factors and coactivators such as CREB and CBP, respectively. Conceptually, these findings are similar to those of our previous study showing that HDAC inhibition enhanced hippocampal synaptic plasticity in a CBP-dependent manner (Vecsey et al., 2007). Inducing a histone hyperacetylated state is not sufficient either to enhance hippocampal synaptic plasticity (Vecsey et al., 2007) or enhance memory for object recognition (this study).

Our finding that the GR, but not MR, is necessary for both corticosterone- and HDAC inhibition-dependent enhancement of long-term memory is similar to results from a recent study examining how novelty stress induces phospho-acetylation of histone H3 via GRs. Novelty stress, induced by varying the light intensity in the experimental room, resulted in light intensitydependent increases in the combined phosphorylation of serine 10 and acetylation of lysine 14 on histone $\mathrm{H} 3$ in the dentate gyrus (Chandramohan et al., 2007). Pretreatment of rats with a GR antagonist nearly abolished the phospho-acetylation of histone H3 following novelty stress (Chandramohan et al., 2007). In contrast, MR antagonists did not modulate the effects of the stress on histone phospho-acetylation. Thus, similar to our findings with regard to memory formation, stress-induced changes in chromatin modification appear to depend on GR, but not MR, activity.

Two findings indicated that CBP, a potent histone acetyltransferase, may be involved in both object recognition and object location memory. First, histone acetylation in both the insular cortex and the hippocampus increased following training. Second, HDAC inhibition enhanced object recognition memory via HDAC inhibition in the insular cortex as well as object location memory via HDAC inhibition in the hippocampus. Indeed, we observed that $24 \mathrm{~h}$ retention of object recognition and object location memory was significantly impaired in genetically modified $\mathrm{Cb} p$ mutant mice (CBP ${ }^{\mathrm{KIX} / \mathrm{KIX}}$ mice) in which both alleles of $C b p$ express mutant protein with a triple point mutation in the phospho-CREB (KIX) binding domain of CBP. Thus, impairing CBP function results in deficits in long-term memory for both object recognition and object location, whereas increasing histone acetylation in a site-specific manner via HDAC inhibition in 
the insular cortex and the hippocampus enhances memory for object recognition and object location, respectively.

Our data demonstrate that for object recognition memory, corticosterone signaling via membrane-bound GRs results in increased CREB phosphorylation and histone acetylation involved in long-term memory formation in the insular cortex. We presumed that the histone acetylation would be performed by CBP as the phosphorylation of CREB induces the interaction between CREB and CBP via the KIX domain of CBP. However, our data indicate that corticosterone enhances long-term object recognition memory independently of CBP. In contrast, the finding that corticosterone failed to enhance object location memory in the $\mathrm{CBP}^{\mathrm{KIX} / \mathrm{KIX}}$ mice suggests that corticosterone enhances object location memory in the hippocampus in a CBP-dependent manner. This interpretation fits well with our previous findings that HDAC inhibition enhances hippocampal synaptic plasticity in a CBP-dependent manner (Vecsey et al., 2007) and that HDAC inhibition enhances memory in $\mathrm{CBP}^{\mathrm{KIX} / \mathrm{KIX}}$ mice for long-term object recognition memory in a CBP-independent manner (Stefanko et al., 2009). Thus, although CBP is required for normal molecular mechanisms underlying long-term memory formation for object recognition and object location, it may be that this condition changes in the presence of modulatory influences such as those resulting from corticosterone or HDAC inhibition in the insular cortex. Further studies are necessary to determine exactly how these modulatory mechanisms enhance memory for object recognition in the insular cortex.

Previous studies have shown that the phosphorylation of CREB is not always sufficient to activate CREB:CBP-mediated gene expression. In cell culture, phosphorylation of CREB at serine 133 (the event triggering $\mathrm{CBP}$ recruitment) can be uncoupled from CREB:CBP-mediated transcription (Chawla et al., 1998; Hardingham et al., 1999). Chawla et al. (1998) proposed a model in which CREB:CBP-mediated gene expression requires two steps: the phosphorylation of CREB (resulting in the recruitment of CBP) and the phosphorylation of CBP for its activation. This model suggests how different signaling cascades may initiate different transcriptional pathways despite converging on a common transcription factor like CREB. Thus, even though corticosterone signaling may result in phosphorylation of CREB at serine 133, such actions may be insufficient to activate CREB: CBP-mediated gene expression in the insular cortex.

In summary, both glucocorticoids and the regulation of histone modifications are well known to enhance memory consolidation of emotionally arousing experiences. The present findings provide evidence that glucocorticoids and histone modification mechanisms interact in enhancing memory consolidation. Thus, these findings indicate that glucocorticoids affect gene expression required for long-term memory consolidation not only via their classic genomic mechanism by serving as transcription factors, but also via the regulation of chromatin structure. This finding is critical as one of the potential features of chromatin modifications is that they may provide transient and potentially stable epigenetic marks in the service of activating and/or maintaining transcriptional processes, which in turn may ultimately participate in the molecular mechanisms required for neuronal changes subserving long-lasting changes in behavior. As an epigenetic mechanism of transcription, chromatin modification has been shown to maintain cellular memory (e.g., cell fate) and may underlie the strengthening and maintenance of synaptic connections required for long-term changes in behavior and may significantly contribute to our understanding of why emotionally arousing experiences are well remembered.

\section{References}

Alarcón JM, Malleret G, Touzani K, Vronskaya S, Ishii S, Kandel ER, Barco A (2004) Chromatin acetylation, memory, and LTP are impaired in $\mathrm{CBP}+/-$ mice: a model for the cognitive deficit in Rubinstein-Taybi syndrome and its amelioration. Neuron 42:947-959.

Balderas I, Rodriguez-Ortiz CJ, Salgado-Tonda P, Chavez-Hurtado J, McGaugh JL, Bermudez-Rattoni F (2008) The consolidation of object and context recognition memory involve different regions of the temporal lobe. Learn Mem 15:618-624.

Barrett RM, Wood MA (2008) Beyond transcription factors: the role of chromatin modifying enzymes in regulating transcription required for memory. Learn Mem 15:460-467.

Beato M, Sánchez-Pacheco A (1996) Interaction of steroid hormone receptors with the transcription initiation complex. Endocr Rev 17:587-609.

Bermúdez-Rattoni F (2004) Molecular mechanisms of taste-recognition memory. Nat Rev Neurosci 5:209-217.

Bermudez-Rattoni F, Okuda S, Roozendaal B, McGaugh JL (2005) Insular cortex is involved in consolidation of object recognition memory. Learn Mem 12:447-449.

Bourtchouladze R, Lidge R, Catapano R, Stanley J, Gossweiler S, Romashko D, Scott R, Tully T (2003) A mouse model of Rubinstein-Taybi syndrome: defective long-term memory is ameliorated by inhibitors of phosphodiesterase 4. Proc Natl Acad Sci U S A 100:10518-10522.

Buchanan TW, Lovallo WR (2001) Enhanced memory for emotional material following stress-level cortisol treatment in humans. Psychoneuroendocrinology 26:307-317.

Chandramohan Y, Droste SK, Reul JM (2007) Novelty stress induces phospho-acetylation of histone $\mathrm{H} 3$ in rat dentate gyrus granule neurons through coincident signalling via the $\mathrm{N}$-methyl-D-aspartate receptor and the glucocorticoid receptor: relevance for c-fos induction. J Neurochem 101:815-828.

Chawla S, Hardingham GE, Quinn DR, Bading H (1998) CBP: a signalregulated transcriptional coactivator controlled by nuclear calcium and CaM kinase IV. Science 281:1505-1509.

Chiyo T, Yamazaki T, Aoshika K, Kominami S, Ohta Y (2003) Corticosterone enhances adrenocorticotropin-induced calcium signals in bovine adrenocortical cells. Endocrinology 144:3376-3381.

Chrivia JC, Kwok RP, Lamb N, Hagiwara M, Montminy MR, Goodman RH (1993) Phosphorylated CREB binds specifically to the nuclear protein CBP. Nature 365:855-859.

de Kloet ER, Oitzl MS, Joëls M (1999) Stress and cognition: are corticosteroids good or bad guys? Trends Neurosci 22:422-426.

de Quervain DJ-F, Roozendaal B, McGaugh JL (1998) Stress and glucocorticoids impair retrieval of long-term spatial memory. Nature 394:787-790.

Fischer A, Sananbenesi F, Wang X, Dobbin M, Tsai LH (2007) Recovery of learning and memory is associated with chromatin remodelling. Nature 447:178-182.

Guan Z, Giustetto M, Lomvardas S, Kim JH, Miniaci MC, Schwartz JH, Thanos D, Kandel ER (2002) Integration of long-term-memory-related synaptic plasticity involves bidirectional regulation of gene expression and chromatin structure. Cell 111:483-493.

Hardingham GE, Chawla S, Cruzalegui FH, Bading H (1999) Control of recruitment and transcription-activating function of CBP determines gene regulation by NMDA receptors and L-type calcium channels. Neuron 22:789-798.

Johnson LR, Farb C, Morrison JH, McEwen BS, LeDoux JE (2005) Localization of glucocorticoid receptors at postsynaptic membranes in the lateral amygdala. Neuroscience 136:289-299.

Kasper LH, Boussouar F, Ney PA, Jackson CW, Rehg J, van Deursen JM, Brindle PK (2002) A transcription-factor-binding surface of coactivator p300 is required for haematopoiesis. Nature 419:738-743.

Korzus E, Rosenfeld MG, Mayford M (2004) CBP histone acetyltransferase activity is a critical component of memory consolidation. Neuron 42:961-972.

Kuhlmann S, Wolf OT (2006) Arousal and cortisol interact in modulating memory consolidation in healthy young men. Behav Neurosci 120:217-223.

Levenson JM, O'Riordan KJ, Brown KD, Trinh MA, Molfese DL, Sweatt JD (2004) Regulation of histone acetylation during memory formation in the hippocampus. J Biol Chem 279:40545-40559. 
McGaugh JL, Roozendaal B (2002) Role of adrenal stress hormones in forming lasting memories in the brain. Curr Opin Neurobiol 12:205-210.

Miranda MI, Quirarte GL, Rodriguez-Garcia G, McGaugh JL, Roozendaal B (2008) Glucocorticoids enhance taste aversion memory via actions in the insular cortex and basolateral amygdala. Learn Mem 15:468-476.

Okuda S, Roozendaal B, McGaugh JL (2004) Glucocorticoid effects on object recognition memory require training-associated emotional arousal. Proc Natl Acad Sci U S A 101:853-858.

Paxinos G, Watson C (2005) The rat brain in stereotaxic coordinates, Ed 4. San Diego: Academic.

Piterkin P, Cole E, Cossette MP, Gaskin S, Mumby DG (2008) A limited role for the hippocampus in the modulation of novel-object preference by contextual cues. Learn Mem 15:785-791.

Quirarte GL, Roozendaal B, McGaugh JL (1997) Glucocorticoid enhancement of memory storage involves noradrenergic activation in the basolateral amygdala. Proc Natl Acad Sci U S A 94:14048-14053.

Reed CL, Shoham S, Halgren E (2004) Neural substrates of tactile object recognition: an fMRI study. Hum Brain Mapp 21:236-246.

Roozendaal B, Nguyen BT, Power AE, McGaugh JL (1999) Basolateral amygdala noradrenergic influence enables enhancement of memory consolidation induced by hippocampal glucocorticoid receptor activation. Proc Natl Acad Sci U S A 96:11642-11647.

Roozendaal B, Quirarte GL, McGaugh JL (2002) Glucocorticoids interact with the basolateral amygdala beta-adrenoceptor-cAMP/PKA system in influencing memory consolidation. Eur J Neurosci 15:553-560.

Roozendaal B, Okuda S, Van der Zee EA, McGaugh JL (2006) Glucocorticoid enhancement of memory requires arousal-induced noradrenergic activation in the basolateral amygdala. Proc Natl Acad Sci U S A 103: 6741-6746.

Roozendaal B, Barsegyan A, Lee S (2008) Adrenal stress hormones, amygdala activation, and memory for emotionally arousing experiences. Prog Brain Res 167:79-97.

Sandi C, Pinelo-Nava MT (2007) Stress and memory: behavioral effects and neurobiological mechanisms. Neural Plast 2007:78970.

Stefanko DP, Barrett RM, Ly AR, Reolon GK, Wood MA (2009) Modulation of long-term memory for object recognition via HDAC inhibition. Proc Natl Acad Sci U S A 106:9447-9452.

Vecsey CG, Hawk JD, Lattal KM, Stein JM, Fabian SA, Attner MA, Cabrera SM, McDonough CB, Brindle PK, Abel T, Wood MA (2007) Histone deacetylase inhibitors enhance memory and synaptic plasticity via CREB: CBP-dependent transcriptional activation. J Neurosci 27:6128-6140.

Wood MA, Kaplan MP, Park A, Blanchard EJ, Oliveira AM, Lombardi TL, Abel T (2005) Transgenic mice expressing a truncated form of CREBbinding protein (CBP) exhibit deficits in hippocampal synaptic plasticity and memory storage. Learn Mem 12:111-119.

Wood MA, Attner MA, Oliveira AM, Brindle PK, Abel T (2006) A transcription factor-binding domain of the coactivator CBP is essential for longterm memory and the expression of specific target genes. Learn Mem 13:609-617.

Yeh SH, Lin CH, Gean PW (2004) Acetylation of nuclear factor-kappaB in rat amygdala improves long-term but not short-term retention of fear memory. Mol Pharmacol 65:1286-1292.

Zheng J, Ali A, Ramirez VD (1996) Steroids conjugated to bovine serum albumin as tools to demonstrate specific neuronal membrane binding sites. J Psychiatry Neurosci 21:187-197. 